\title{
Understanding the patterns of spread of HIVIAIDS in Nigeria: a systematic review and meta-analysis of primary research articles
}

\author{
Abstract \\ Objectives: \\ a) To identify key themes/variables in HIV/AIDS epidemiology in Nigeria \\ b) To highlight regional variations in HIV/AIDS epidemiology in Nigeria \\ c) To identify common interventions for HIV/AIDS in Nigeria \\ d) To provide information useful for research
}

Methods: Systematic review and meta-analysis of primary research articles from Nigeria.

Results: HIV/AIDS epidemic in Nigeria is currently a mixed epidemic perceived and understood differently across the country. Variables that shape the epidemiology include stigma, inaccurate knowledge about HIV/AIDS, cultural norms, religion, gender, poor risk perception, poverty and the lack of a robust and well organised response. Gender (OR 1.95, $95 \%$ CI 1.27 to 3.02 , p-value $<0.00001$ ) and history of sexually transmitted infections (OR $2.19,95 \%$ CI 1.99 to 2.41 , p-value $<0.00001$ ) were significantly associated with increased risk for HIV/AIDS

Conclusion: There is need for evidence based and focused public policies as well as robust and comprehensive intervention approaches in Nigeria. In addition, HIV/AIDS research in Nigeria should be targeted at unravelling the mechanisms through which risk factors work to produce increased HIV/AIDS prevalence.

Keywords: HIV/AID, Nigeria, Prevalence, Systematic review, risk factors, epidemiology
Volume 2 Issue 4 - 2015

\author{
Oono IP,' Ong E, ${ }^{2}$ Shahaduz Z,' Pearce MS' \\ 'Institute of Health and Society, Newcastle University, United \\ Kingdom \\ 2Department of Infection \& Tropical Medicine, Royal Victoria \\ Infirmary Newcastle Upon Tyne NHS foundation Trust, United \\ Kingdom
}

Correspondence: Oono Inalegwu, Institute of Health and Society, Newcastle University, Baddiley-Clark building. Newcastle Upon Tyne, NE2 4AX, Email i.p.oono@newcastle.ac.uk

Received: March 17,2015 | Published: April 23, 2015
Abbreviations: WHO, World Health Organization; WoK, Web of Knowledge

\section{Introduction}

HIV/AIDS remains a global challenge, claiming more than 39 million lives since it was first discovered. ${ }^{1}$ The burden of HIV/AIDS is greatest in sub-Saharan Africa where an estimated 24.7 million children and adults live with HIV/AIDS today (worldwide there are a total of 35 million adults and children living with HIV/AIDS). ${ }^{2}$ The high burden of HIV/AIDS in sub-Saharan Africa has serious negative consequences for economies in this region as HIV/AIDS disproportionately affects the productive age group. ${ }^{3}$ Furthermore, high HIV/AIDS prevalence places enormous pressures on healthcare which is already stretched and in short supply. A clear understanding of the way local factors work to promote or halt the spread of HIV/ AIDS in sub-Sahara of Africa will form the foundation for effective intervention programs and strengthen the global response to HIV/ AIDS. In this review we focus on Nigeria, a country in the sub-Sahara. This is because Nigeria is the most populous country in Africa, with a population of over 160 million people ${ }^{4}$ and has a significant burden of HIV/AIDS. Effective control of HIV/AIDS in Nigeria has huge implications for the West African sub-region.

\section{Methods}

The PRISMA checklist for the reporting of systematic reviews was used for the reporting. The protocol for this research was published on PROSPERO. ${ }^{5}$ The following databases were searched for relevant articles: MEDLINE; UNAIDS; World Health Organization (WHO); EMBASE; EBM reviews; Cochrane Central Register of Controlled Trials (CENTRAL); PsycINFO; ProQuest (all databases); Scopus; Web of Knowledge (WoK); Google Scholar; Journal of Public health in Africa; African journal of Infectious diseases; African journal of AIDS research; CINAHL (EBSCO); Global health abstract; PubMed; AIDS inline. Reference lists of relevant publications were also searched and experts were contacted, where necessary, during the search. The following restrictions were applied to the search: studies published between 2003 and2014; English language only; adults only.

The last search was conducted in the last week of November 2014. Please see Table 1 below for sample search terms. Adaptations of these terms were used on other databases listed above. Relevant publications were identified and included in the review. Publications included were those that included Nigerian participants, regardless of where they were conducted. A piloted data extraction form designed by one of the authors (OIP) was used for the data extraction process and where relevant primary research authors were contacted for missing data. Variables of interest and details about the inclusion criteria are available in the published protocol. ${ }^{5}$ A number of appraisal tools were used in quality assessment process ${ }^{6-8}$ and no study was excluded on the basis of their quality ratings. Information gathered from the appraisal exercise only serves to provide a statement of confidence about the research findings. Random effects meta-analyses was used to pool estimates of risk in the studies reviewed and heterogeneity among the studies was quantified using $I 2$ values. 
Table I Sample search strategy (Medline)

I. HIV.mp. [mp=title, abstract, subject headings, heading word, drug trade name, original title, device manufacturer, drug manufacturer, device trade name, keyword]

2. HIV infection*.mp. [mp=title, abstract, subject headings, heading word, drug trade name, original title, device manufacturer, drug manufacturer, device trade name, keyword]

3. Human immun*deficiency virus.mp. [mp=title, abstract, subject headings, heading word, drug trade name, original title, device manufacturer, drug manufacturer, device trade name, keyword]

4. Acquired Immun*deficiency Syndrome.mp. [mp=title, abstract, subject headings, heading word, drug trade name, original title, device manufacturer, drug manufacturer, device trade name, keyword]

5. I or 2 or 3 or 4

6. Stigma.mp. [mp=title, abstract, subject headings, heading word, drug trade name, original title, device manufacturer, drug manufacturer, device trade name, keyword]

7. Telephone.mp. [mp=title, abstract, subject headings, heading word, drug trade name, original title, device manufacturer, drug manufacturer, device trade name, keyword]

8. Social marketing.mp. [mp=title, abstract, subject headings, heading word, drug trade name, original title, device manufacturer, drug manufacturer, device trade name, keyword]

9. (Voluntary counselling and testing).mp. [mp=title, abstract, subject headings, heading word, drug trade name, original title, device manufacturer, drug manufacturer, device trade name, keyword]

10. Voluntary counselling.mp. [mp=title, abstract, subject headings, heading word, drug trade name, original title, device manufacturer, drug manufacturer, device trade name, keyword]

II. Knowledge.mp. [mp=title, abstract, subject headings, heading word, drug trade name, original title, device manufacturer, drug manufacturer, device trade name, keyword]

12. Internet.mp. [mp=title, abstract, subject headings, heading word, drug trade name, original title, device manufacturer, drug manufacturer, device trade name, keyword]

13. Behavio*ral.mp. [mp=title, abstract, subject headings, heading word, drug trade name, original title, device manufacturer, drug manufacturer, device trade name, keyword]

14. Behavio*r.mp. [mp=title, abstract, subject headings, heading word, drug trade name, original title, device manufacturer, drug manufacturer, device trade name, keyword]

15. School.mp. [mp=title, abstract, subject headings, heading word, drug trade name, original title, device manufacturer, drug manufacturer, device trade name, keyword]

16. Community.mp. [mp=title, abstract, subject headings, heading word, drug trade name, original title, device manufacturer, drug manufacturer, device trade name, keyword]

17. Information communication.mp. [mp=title, abstract, subject headings, heading word, drug trade name, original title, device manufacturer, drug manufacturer, device trade name, keyword]

18. Home.mp. [mp=title, abstract, subject headings, heading word, drug trade name, original title, device manufacturer, drug manufacturer, device trade name, keyword]

19. Economic.mp. [mp=title, abstract, subject headings, heading word, drug trade name, original title, device manufacturer, drug manufacturer, device trade name, keyword]

20. Abstinence.mp. [mp=title, abstract, subject headings, heading word, drug trade name, original title, device manufacturer, drug manufacturer, device trade name, keyword]

2I. Abstinence plus.mp. [mp=title, abstract, subject headings, heading word, drug trade name, original title, device manufacturer, drug manufacturer, device trade name, keyword]

22. Mass media.mp. [mp=title, abstract, subject headings, heading word, drug trade name, original title, device manufacturer, drug manufacturer, device trade name, keyword]

23. Skill* building.mp. [mp=title, abstract, subject headings, heading word, drug trade name, original title, device manufacturer, drug manufacturer device trade name, keyword]

24. Psychosocial.mp. [mp=title, abstract, subject headings, heading word, drug trade name, original title, device manufacturer, drug manufacturer, device trade name, keyword]

25. Condom.mp. [mp=title, abstract, subject headings, heading word, drug trade name, original title, device manufacturer, drug manufacturer, device trade name, keyword]

26. Campaign*.mp. [mp=title, abstract, subject headings, heading word, drug trade name, original title, device manufacturer, drug manufacturer, device trade name, keyword]

27. 6 or 7 or 8 or 9 or 10 or 11 or 12 or 13 or 14 or 15 or 16 or 17 or 18 or 19 or 20 or 21 or 22 or 23 or 24 or 25 or 26

28. Succes*.mp. [mp=title, abstract, subject headings, heading word, drug trade name, original title, device manufacturer, drug manufacturer, device trade name, keyword]

29. Gain*.mp. [mp=title, abstract, subject headings, heading word, drug trade name, original title, device manufacturer, drug manufacturer, device trade name, keyword] 
Table Continued...

30. Progress*.mp. [mp=title, abstract, subject headings, heading word, drug trade name, original title, device manufacturer, drug manufacturer, device trade name, keyword]

3I. Change*.mp. [mp=title, abstract, subject headings, heading word, drug trade name, original title, device manufacturer, drug manufacturer, device trade name, keyword]

32. Mortalit*.mp. [mp=title, abstract, subject headings, heading word, drug trade name, original title, device manufacturer, drug manufacturer, device trade name, keyword]

33. Incidence.mp. [mp=title, abstract, subject headings, heading word, drug trade name, original title, device manufacturer, drug manufacturer, device trade name, keyword]

34. Prevalence.mp. [mp=title, abstract, subject headings, heading word, drug trade name, original title, device manufacturer, drug manufacturer, device trade name, keyword]

35. Problem*.mp. [mp=title, abstract, subject headings, heading word, drug trade name, original title, device manufacturer, drug manufacturer, device trade name, keyword]

36. Limitation*.mp. [mp=title, abstract, subject headings, heading word, drug trade name, original title, device manufacturer, drug manufacturer, device trade name, keyword]

37. Set*back*.mp. [mp=title, abstract, subject headings, heading word, drug trade name, original title, device manufacturer, drug manufacturer, device trade name, keyword]

38. Politic*.mp. [mp=title, abstract, subject headings, heading word, drug trade name, original title, device manufacturer, drug manufacturer, device trade name, keyword]

39. Economic.mp. [mp=title, abstract, subject headings, heading word, drug trade name, original title, device manufacturer, drug manufacturer, device trade name, keyword]

40. 28 or 29 or 30 or 31 or 32 or 33 or 34 or 35 or 36 or 37 or 38 or 39

4I. 41.5 and 27 and 40

42. Exp Africa,Western/ or exp Africa, Northern/ or exp South Africa/ or exp Africa, Eastern/ or Africa.mp. or exp "Africa South of the Sahara"/ or $\exp$ Africa, Central/ or exp Africa/ or exp Africa, Southern/

43. $4 \mathrm{I}$ and 42

Two forms of data were encountered in this review - qualitative and quantitative data. Qualitative data were analysed thematically and the findings included in the narrative synthesis. Quantitative data were included in the meta-analyses. Details of these analyses (qualitative and quantitative) and a list of articles included in the review will be published in a separate document. Data were managed and analysed using End Note X7, NVIVO 10, Rev Man 5.3 and survey monkey software packages respectively. ${ }^{9-12}$

\section{Results}

Ninety-three publications relevant to Nigeria were identified from the databases and reference lists search and were thus included in the review. A list of studies included in the review as well as relevant figures will be published in a separate document as supplementary data.

\section{Study selection}

A total of 15,578 publications were identified through databases search. Another one hundred and ninety-three records were identified through searching of reference lists of relevant articles (Figure 1)

\section{Study characteristics}

Most of the publications (84) are cross sectional. There were six case-control studies, two cohort studies and one randomised controlled trial. Eight of the publications were qualitative; ten were mixed methods and 75 were quantitative. All of the studies reviewed included Nigerian participants except for two (Abiona et' al. and Nwauche) in which other nationals and migrant populations were included alongside Nigerians. Most of the studies were carried out in multiple locations within Nigeria: the North East in 12 publications; the North West in 18 publications; the Middle Belt (North Central) in 19 publications; South South in 20 publications; South East in 23 publications and South West in 44 publications. Two of the publications analysed their data using "North" and "South" regional designations. Six publications used data from nationally representative databases. Research participants were predominantly in the second through to the fourth decades of life. Eighty-five of the articles reviewed were published between 2005 and 2013 .

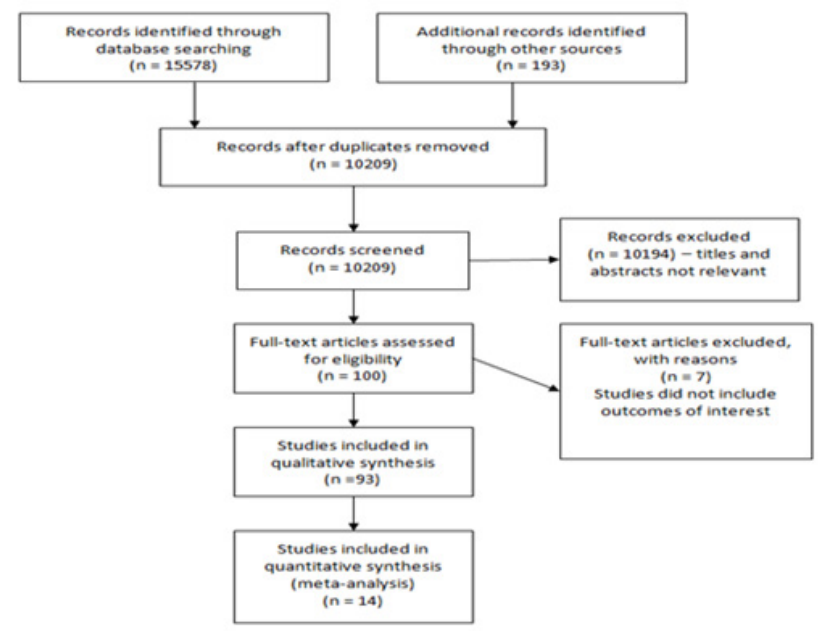

Figure I Study flow diagram.

Fifty-one of the reviewed publications recruited from the general public, ten recruited people living with HIV/AIDS, five recruited uniform personnel, four recruited men who have sex with men, four recruited traditional birth attendants/traditional healers, three recruited long distance truck drivers/motor park workers, three recruited female commercial sex workers and brothel workers, two recruited health workers (hospital and dental staff), two recruited injecting drug users, two recruited religious leaders and key local figures, one had a mixed group (general public, brothel sex workers, sero discordant patients, 
taxi drivers etc.), one recruited trainee sailors, one had media staff as participants, one recruited migrant workers, one had prison inmates and one had secondary school teachers.

\section{The quality assessment exercise}

Based on our assessment, most of the studies reviewed were of low quality grading, despite all being published in peer reviewed journals. Important setbacks observed in the quality assessment process were largely in the methods sections (poorly defined methods for selection of participants, poor reporting, small numbers, lack of description of tools used for data collection etc.). While most of the publication findings may be accurate for the local populations included in the studies, generalisation beyond these populations is seriously limited.

\section{The narrative synthesis}

Factors highlighted in Figure 2 below are the most frequently occurring themes (with themes in larger text fonts occurring more frequently). Themes around low rates of condom use featured very strongly in the publications reviewed. The main reason for low patronage of condoms appears to be the perceived societal stigma associated with condom use - it is largely seen as a confirmation of being lose and immoral. This stigmatization takes place despite adequate knowledge of the protection condoms could provide against HIV/AIDS. The quote below demonstrates how a girl who keeps condoms could be viewed as a prostitute.

\section{Gender condom Knowhow Risk Perception Long Absence Stigma Age Younger LoW Condom Treatment Access $_{\text {S }}$ Inaccurate Knowledge ossossue MSP Education}

Figure 2 Word cloud showing some important risk factors for HIV/AIDS spread in Nigeria based on frequency of occurrence (words in bigger fonts occur more frequently).

Gender: Gender Inequality; Condom Knowhow: Poor Knowledge of How to Use Condoms; Risk Perception: Poor Risk Perception; Long Absence: Long Stay Away From Home; Stigma: Stigma From HIV/AIDS; Age Younger: Younger Age Associated With Greater Odds For HIV/AIDS Infection; Low Condom: Low Condom Use Rates; Treatment Access: Factors That Hinder Access to Treatment; Inaccurate Knowledge: Incorrect Knowledge About HIV/AIDS; Disclosure: Non-Disclosure of HIV/AIDS Status By HIV+ individuals; MSP: Multiple Sex Partners; Education: Low Level of Educational Attainment

"If a girl keeps a condom in her room you will feel somehow, you know, like she is a professional [prostitute]" a participant in the Smith study. ${ }^{13}$

Poor knowledge on how to correctly use male condoms and availability of condoms are also factors that limit condom use in Nigeria. Other factors that influence condom use include the perception that condoms are a barrier to relationship intimacy and fatalistic tendencies where clients of sex workers would go the extra mile to convince sex workers not to use condoms during sex, claiming "love" and being "boyfriends" as legitimate reasons not to use condoms.
"He said 'Don't you love me? I love you and know you're clean. I believe you don't have anything in your body. I trust you ...don't you love me ... you don't love me? I'm not your client now, I'm your boyfriend ... how can we get married if you continue like this?' Sometime we use condom, but most time we don't. I love him and I don't want this work all my life" participant quote in Munoz et al. ${ }^{14}$ study.

Stigma is another important variable in HIV/AIDS epidemiology in Nigeria. Stigma associated with HIV/AIDS as well as stigmatisation of people living with HIV/AIDS was described in a number of the publications reviewed. Stigmatisation of HIV/AIDS occurs at different levels within the Nigerian society and plays a huge role in influencing peoples' decisions about utilisation of screening and treatment services. Stigmatisation is often encountered at family and community levels, making it difficult for people who live with HIV/ AIDS to be accepted in their communities. In one study, the media have been implicated in stigmatisation of people living with HIV/ AIDS in Nigeria.

"“"They [journalists] contributed to this problem. The way they narrated the story of HIV when it started and the picture they showed us still scare us and these have led to the stigma. Before we can accept persons living with HIV/AIDS fully, the media should un-do the harm they have created...." A traditional ruler in Odimegwu et al. ${ }^{15}$ study.

"If the girls knew ... they would make me die fast. They would kill me before my time. Instead of help they would scandalize the matter. They would stop communicating with me. When HIV comes your friend becomes your enemy. I will be in danger for my life if they know" participant in Munoz et al. ${ }^{14}$ study.

"Stigmatization can lead to concealment of status so that people will not know. This will deny early medical attentions. Concealment of status is one of the reasons why the infection cannot be controlled. A lot of people have the disease but do not want to declare publicly their status for these fears" participant in Odimegwu et al. ${ }^{15}$ study.

"A female opinion leader also indicated that it is difficult to even come near PLHIV [people living with HIV], let alone trying to help them. If my family member is living with HIV/AIDS, I would never mention it to anybody outside the family. This is because it is a big shame for all the members of the family. According to her, it is a big shame for the family that one of their own is suffering from a disease of immoral people meaning that the entire family members are likely to be immoral people" participant response in Muoghalu and Jegede ${ }^{16}$ study.

Inaccurate knowledge, rather than outright lack of knowledge, seems to be the more important variable when knowledge of HIV/ AIDS was explored in the publications reviewed. Those who demonstrated outright lack of knowledge, or very poor knowledge of HIV/AIDS prevention, were traditional healers (TH) and traditional birth attendants (TBA). The general public on the other hand failed to recognise the heterosexual contact as the prevailing means through which HIV/AIDS is contracted - they will rather cite the use of unsterilized materials as being more important in HIV/AIDS spread. Another ominous trend is that knowledge did not seem to translate into action in most of the studies reviewed.

"While both male and female migrants have high levels of knowledge about HIV/AIDS, common responses also suggest some misconceptions about modes of transmission. For example, 46 percent of respondents in Kano and 43 percent in Aba identified sharing barbing instruments (like razors and scissors) as a mode of 
transmission. While this mode of transmission cannot be ruled out these figures reinforce anecdotal data that I collected in numerous discussions about AIDS. These conversations suggest that young people's awareness that HIV can be contracted via sexual intercourse is not the same as recognition that heterosexual intercourse is by far the most likely mode of transmission in Nigeria. I was struck by how frequently migrants said that one of the main ways they protect themselves against AIDS is by having their own barbing and shaving instruments" Smith ${ }^{17}$ study.

"Although a good proportion of HIV-positive women were aware that unprotected sex could expose individuals to HIV infection, yet, more than half of them still engaged in unprotected sex showing that knowledge of HIV transmission is not a guarantee to encourage HIV prevention" Enwerej $1^{18}$ study.

"HIV can be transmitted through razor blades....you can catch it through a syringe.' 'Like I said earlier, it has no cure, that's why I am worried" Adebayo et al. ${ }^{19}$ study.

Poor risk perception is another important variable in the epidemiology of HIV/AIDS in Nigeria. Poor risk perception, in this case, refers to a phenomenon in which individuals who engage in activities that are known to be "high risk" for contracting HIV/ AIDS hold the view that they are at little or no risk for contracting HIV/AIDS. Those caught in this web of poor risk perception tend to project the risk of contracting HIV/AIDS infection onto "immoral others". Religion, as well as fatalistic beliefs or tendencies, appear to be important drivers for this behaviour.

"I am not at risk, because if it is one girlfriend that will lead to the risk, but trust and love in her is what is making me say so. Keeping one girlfriend to me is not risky, but keeping [many of] them at random is a big risk"Adebayo et al. ${ }^{19}$

"Almost all the respondents (96.4\%) knew themselves to be at high risk of contracting STDs, while $87.6 \%$ felt that it was impossible for them to catch AIDS" Ekanem et al. ${ }^{20}$

Furthermore, "multiple sexual partnerships" is another key variable in the spread of HIV/AIDS in Nigeria. This factor is of paramount importance when viewed in a setting where HIV/AIDS prevalence is high and condom use is low. Females have a disproportionately greater risk for contracting HIV/AIDS compared to their male counterparts. While it is well documented that the female biology could explain the increased risk for HIV/AIDS, behavioural factors that may play a part in this include the widely held view that the role of females in relationships is passive, a norm that impacts negatively on the capacity of females to negotiate safer options in sexual relationships.

"A woman is expected to obey her husband whatever the circumstance" a Muslim woman leader, in Saddiq et al. ${ }^{21}$

Based on the reviewed publications, age is a risk factor for HIV/ AIDS as it disproportionately affects people in the younger age group -predominantly those in the age range of 18-40 years. However, one studies (Adeyemo et al. ${ }^{22}$ found that the prevalence of HIV/AIDS was higher in pregnant women above 40 years of age.

Long absence from home, frequent travel and movement of people are important factors in the spread of HIV/AIDS in Nigeria. This is especially important for specific groups such as uniformed personnel and long distance truck drivers who share a common denominator -long stays away from home and staying in different cities. The risk for intra-city transport workers, on the other hand, stems from lack of adequate knowledge and high risk sexual behaviours. Six of the papers reviewed suggested that movements of people from one place to another (between high prevalence and low prevalence areas) play an important role in the spread of HIV/AIDS in Nigeria. High risk sexual behaviour observed among migrant oil workers compared to the local population, in one of the studies reviewed highlights the role of migrants in HIV/AIDS epidemic in Nigeria.

As regards MSM (men who have sex with men, a high risk group) important variables for HIV/AIDS epidemiology includes high mobility, differential HIV/AIDS prevalence among MSM in different States, inconsistent condom use and links with female sex partners (unprotected sex with sex workers and non-members of the MSM community). These variables have the potential for further HIV transmission within MSM networks, and between MSM and the general population.

Prompt and uninterrupted access to medications for people living with HIV/AIDS has been shown to reduce the risk for HIV/AIDS transmission. Issues relating to access to treatment for people living with HIV/AIDS play a vital role in the spread of HV/AIDS in Nigeria. Treatment centres are mostly situated in urban centres (where those in the higher wealth strata reside). This skew in services poses serious access challenges for those who reside in rural or sub-urban areas (where the vast majority of those in the lower socioeconomic strata reside). Discrimination, poverty and fears of discontinued funding for medications also play key roles in influencing access to treatment for people living with HIV/AIDS. Another key player in the spread of HIV/AIDS in Nigeria is the willingness of people living with HIV/ AIDS to disclose their status to their partners. This is largely due to the fear of stigmatization that may follow disclosure.

"I am worried because I have multiple sexual partners and the worse thing is that even if your partner is infected, she won't tell you until she pass[es] the disease to you". ${ }^{19}$

Low sexually transmitted diseases detection and treatment rates is another factor that readily promote the spread of HIV/AIDS in Nigeria. Injecting drug users also play important roles in the spread of HIV/AIDS in Nigeria though only few studies were found that looked into trends among this high risk group. The role of three very important factors (religion, culture, and government policies)in shaping HIV/AIDS spread in Nigeria cannot be overlooked. Though less encountered in the reviewed articles, they play hugely important roles in HIV/AIDS epidemiology in Nigeria as they form the context within which most of the risk factors described above operate, shaping the way individuals perceive and therefore, respond to the HIV/AIDS epidemic. Faith-based organisations, being scantily accepting of measures for prevention of HIV/AIDS transmission such as the use of condoms, play a huge limiting role in the containment of HIV/AIDS epidemic in Nigeria.

Abstinence is the preferred method in churches. Condoms and being faithful to a partner are, therefore, reserved for those in marriage. Similarly, Muslim clerics are less likely to be accepting of premarital HIV/AIDS screening, in the North of Nigeria where religion features very strongly in the variables that influence uptake of voluntary counselling and testing for HIV/AIDS..$^{23,24}$ It is a widely held norm in most parts of Nigeria that issues relating to sexuality are private and discussions surrounding sexuality ought to be done in private. Similarly, the culturally acceptable practice of polygamy, sometimes informed by religion (for example Islam practised largely in the North of Nigeria) plays a rather indirect and hugely debatable role in the spread of HIV/AIDS. ${ }^{21,25}$ Some quotes, obtained from a few of the reviewed publications, demonstrating how religion and culture may influence HIV/AIDS spread in Nigeria are included below: 
"Polygamy predisposes to promiscuity as it will be difficult for the man to take care of many women in all aspects, financial, sexual and any other. If the women are not taken care of, they will take care of themselves from outside. This will therefore increase the probability of spreading the disease" a Christian minister in Saddiq et al. ${ }^{21}$ study. "Because women's desire for sex is less than that of men, they can be patient enough to be satisfied in a polygamous home" male Muslim leader in Saddiq et al. ${ }^{21}$

"... Well, you have to understand that this issue is not about what I think about their private lives. I teach them biology, and that's what I am paid to teach them. When we learn blood circulation, we also study about functions of blood and about blood transfusion. I teach them the importance of screening of blood before transfusion, and I use the opportunity to teach them about safe use of needles. We not only study about HIV/AIDS prevention but we also study hepatitis B prevention through safe blood transfusion" Oshi et al. ${ }^{26}$

"Secondary school students can definitely get infected with HIV virus. And they need to be taught how to protect themselves. But what will be the fate of the person who does the teaching? If I try it, I will face serious consequences from my community immediately they get to know". "In my wildest imaginations, I can't even try it. I just can't. My church will send me packing" Oshi et al. ${ }^{26}$

In Nigeria, it is illegal to identify as a homosexual or commercial sex worker. These policies and legal requirements drive homosexuals underground and make commercial sex workers vulnerable to abuse and exploitation. It also encourages unsafe work environments for commercial sex workers through a loosely regulated sex industry, which, in turn promotes the spread of HIV/AIDS.

"Police say our work is illegal ... that we should go find other jobs. Tell me, is there anyone who wants to do this kind of work all their lives? Many times, when they come here [to raid] they force sex [rape] on us like we are dogs or beat us so much we need to call a doctor. The Police do so many bad things to us, things that are against God's way and what happens? Not a thing. When they force sex, they won't use condom. They bring AIDS to us and then say we give it to them". "As we speak, some places [brothel] do not get this kind of trouble because they are protected ... they pay protection money. Those who don't pay are the ones in a difficult situation as they are raided all the time. It is better to pay the money and do your business in peace; otherwise, clients will even be afraid to come here" Munozi et al. ${ }^{14}$

\section{Interventions encountered in reviewed studies}

Only seven of the publications reviewed explored effectiveness of some commonly used interventions for HIV/AIDS in Nigeria. HIV/AIDS risk reduction model underpinned most of the interventions assessed in the publications. Interventions featured were predominantly behavioural change interventions with one social marketing intervention and one structural intervention.

\section{Meta-analysis results}

\section{Risk of HIVIAIDS based on gender and history of STDs}

Random effects meta-analysis of data from 8 publications recruiting a total of 40095 participants, revealed an increased risk for HIV/AIDS associated with the female gender compared with their male counterpart (OR $1.95,95 \%$ CI 1.27 to 3.02 , p-value < $0.00001, I 2=85 \%$ ). Please see Figure 3 below. Similarly, random effects meta-analysis of data from 2 publications including a total of 24078 participants, revealed increased risk for HIV/AIDS among those with a history of STDs when compared with those who did not have a previous history of STDs (OR 2.19, $95 \%$ CI 1.99 to 2.41 , p-value $<0.00001, I 2=0 \%$ ).

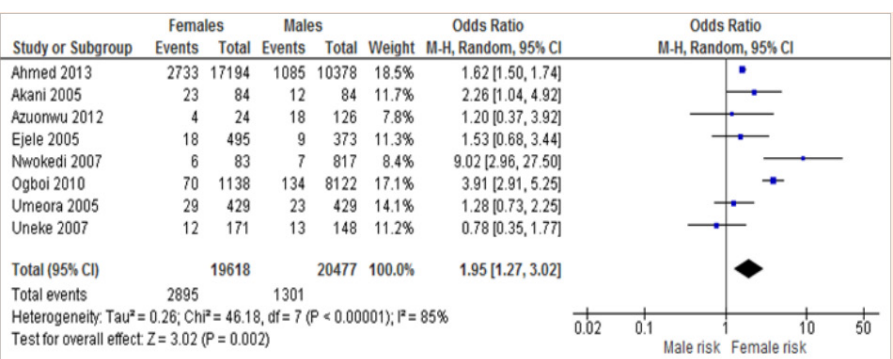

Figure 3 Meta-analyses exploring risk of HIVIAIDS by gender (male vs female).

\section{Risk of HIVIAIDS based on levels of education, age and marital status}

In the random effects meta-analyses exploring some variables of interest (education, age and marital status, we found no statistically significant trend in the risk for HIV/AIDS. Primary education or less versus secondary and above: 5 publications recruiting a total of 2630 participants (OR 1.02, 95\% CI 0.64 to 1.63 , p-value $=0.93, I 2=$ $69 \%$ ). Participants 25 years and below versus those above 25 years: 11 publications recruiting a total of 41032 participants (OR $0.85,95 \%$ CI 0.65 to $1.11, \mathrm{p}$-value $=0.24, I 2=55 \%$ ). Singles versus married: 4 publications recruiting a total of 1222 participants (OR 1.88, 95\% CI 0.48 to 7.43 , p-value $=0.37, I 2=79 \%$ ).

\section{Discussion}

\section{Summary of evidence}

Some key players in the HIV/AIDS epidemic in Nigeria are stigma, inaccurate knowledge about HIV/AIDS, cultural norms, religion, gender issues (in particular the passive role of females in negotiating safer sex), poor risk perception, poverty and the lack of a robust and well organised response. The picture is one of an epidemic which is perceived and understood differently across Nigeria. It is, therefore, plausible to view the HIV/AIDS response in Nigeria as one in which individuals are at different rungs of the behavioural change ladder, as described in the "trans theoretical" model of behaviour change(TTM). ${ }^{27,28}$

There is currently no cure for HIV/AIDS. The mainstay for prevention, therefore, remains treatment for those already infected and prevention for those not infected (the latter heavily influenced by behaviours of individuals and the choices they make). Variables like poverty, poor access to medications and the lack of a robust intervention impacts negatively on availability of treatment for those who are already infected with HIV/AIDS. Central to most models for behaviour change, such the TTM, is the understanding that individuals who have rightly perceived their risks for a particular illness (HIV/ AIDS) are most likely to engage in healthy behaviours relevant for halting the spread of such disease condition. As a result, factors such as stigmatisation, negative cultural norms, poor risk perception, inaccurate knowledge, risk projection to immoral others and religion that have the capacity to shape how one may perceive and internalise their risks for HIV/AIDS do have important implications for HIV/ AIDS epidemiology in Nigeria. The exact mechanism through which these factors operate to influence HIV/AIDS epidemic in Nigeria remains debatable. However, there is no doubt about the limiting role they play in the fight against HIV/AIDS in Nigeria.

There are no huge variations in the types of variables that drive HIV/AIDS in different regions of Nigeria. However, there is enough 
evidence suggesting there are significant variations in the mechanism through which each of these variables impact on HIV/AIDS prevalence. For example religion and culture are two key variables that shape the HIV/AIDS epidemic in Nigeria. The predominantly Muslim north of Nigeria is less receptive of measures like premarital HIV/AIDS screening and condoms (the later seen as a restriction to conception). Whereas, in the largely Christian south, abstinence is the most preferred prevention option by church leaders who consider other HIV prevention options such as "being faithful to a partner and condoms" as exclusive rights for those in marriage.

Interventions commonly used in Nigeria are predominantly directed at risk factors for HIV/AIDS. However, other significant components of effective interventions such as social support for people living with HIV/AIDS, biomedical approaches (such as the use of antiretroviral medications or prevention of HIV/AIDS) and public health policies that promote healthy behaviours (for example policies that criminalises reckless or intentional transmission of HIV/AIDS criminal transmission of HIV/AIDS) are almost non-existent. In fact, prompt and timely commencement of antiretroviral medications has been shown to bring about stigma reversal. ${ }^{29}$ Finally, the interventions are hugely reliant on behavioural change communication models, "education for change". In health behaviour psychology, it is a wellknown fact that education does not always lead to behaviour change.

\section{Limitations}

This review utilised data that were collected, analysed and published by other researchers. There are significant heterogeneity between the studies aggregated, a factor that could have been responsible for us not being able to demonstrate any relationship between risk for HIV/ AIDS and some variables like education, age (25 years and below versus above 25) and marital status. Further statistical analyses (not done in this review) may be needed to explore these variables.

\section{Conclusion and implications for future research and policy making}

There is urgent need for evidence based and focused public policies as well as robust and comprehensive intervention approaches that encourage individuals in the right direction. Research should be targeted at unravelling mechanisms through which risk factors work to produce increased prevalence, especially for pockets of communities where HIV/AIDS still remain significantly higher than the national average.

\section{Research funding}

This research is funded by a Commonwealth Scholarships grant awarded to the first author by the Commonwealth Scholarships Commission in the United Kingdom.

\section{Acknowledgments}

None.

\section{Conflicts of interest}

None.

\section{References}

1. World Health Organization. Fact sheet on HIV. 2013.

2. World Health Organization. Global epidemiology slides. 2013.

3. Dixon S, McDonald S, Roberts J. The impact of HIV and AIDS on Africa's economic development. BMJ. 2002;324(7331):232-234.
4. National Population Commission Nigeria. Nigeria over 167 million population: implications and Challenges. 2013.

5. Inalegwu O, Ong Edmund, Zaman Shahaduz, et al. Understanding the patterns of spread of HIV/AIDS in Nigeria: a review of primary research publications. PROSPERO International prospective register of systematic reviews. 2014.

6. GA Wells, B Shea, D O'Connell, et al. The Newcastle-Ottawa Scale (NOS) for assessing the quality of nonrandomised studies in metaanalyses. Clinical Epidemiology.

7. Higgins JPT, Sally Green. Cochrane Handbook for Systematic Reviews of Interventions Version 5.0.2 The Cochrane Collaboration. 2009

8. National Collaborating Centre for Environmental Health. Critical appraisal of Cross-sectional studies: an adaptation of Newcastle-Ottawa scale and critical appraisal skills prgramme. from http://www.ncceh.ca/ en/practice_policy/evidence_review_process. 2011.

9. Thomson Reuters. Endnote X7 for Windows. 2013.

10. QSR International Pty Ltd. $N$ Vivo qualitative data analysis software. 2014.

11. The Nordic Cochrane Centre the Cochrane Collaboration. Review Manager (RevMan), version 5.3. 2014.

12. Ryan Finley, C Finley. Survey monkey: an online survey service. 2015.

13. Smith DJ. Youth, sin and sex in Nigeria: Christianity and HIV/AIDSrelated beliefs and behaviour among rural-urban migrants. Culture, health and sexuality. 2004;6(5):425-437.

14. Munoz J, Adedimeji A, Alawode O. They bring AIDS to us and say we give it to them': Socio-structural context of female sex workers' vulnerability to HIV infection in Ibadan, Nigeria. Sahara J. 2010;7(2):52-61.

15. Odimegwu C, Adedini SA, Ononokpono DN. HIV/AIDS stigma and utilization of voluntary counselling and testing in Nigeria. BMC Public Health. 2013;13:465.

16. Muoghalu CO, Jegede SA. Perception of HIV/AIDS among the Igbo of Anambra State, Nigeria. Sahara J. 2013;10(1):42-54.

17. Smith DJ. Imagining HIV/AIDS: morality and perceptions of personal risk in Nigeria. Med Anthropol. 2003;22(4):343-372.

18. Enwereji EE. Sexual behaviour and inheritance rights among HIV-positive women in Abia State, Nigeria. Tanzan J Health Res. 2008;10(2):73-78

19. Adebayo SB, Anyanti J, Ankomah A, et al. Understanding self-appraisal of HIV-infection risk among young adults in Nigeria: evidence from a national survey. Afr J AIDS Res. 2010;9(1):51-61.

20. Ekanem EE, Afolabi BM, Nuga AO, et al. Sexual behaviour, HIVrelated knowledge and condom use by intra-city commercial bus drivers and motor park attendants in Lagos, Nigeria. Afr J Reprod Health. 2005;9(1):78-87.

21. Saddiq A, Tolhurst R, Lalloo D, et al. Promoting vulnerability or resilience to HIV? A qualitative study on polygamy in Maiduguri, Nigeria. AIDS Care. 2010;22(2):146-151.

22. Adeyemo BO, Gayawan E, Olusile AO, et al. Prevalence of HIV infection among pregnant women presenting to two hospitals in Ogun state, Nigeria. HIV \& AIDS Review. 2014;13(3):90-94.

23. Nwachukwu CE, Odimegwu C. Regional patterns and correlates of HIV voluntary counselling and testing among youths in Nigeria. Afr J Reprod Health. 2011;15(2):131-146.

24. Umar SA, Oche OM. Knowledge of HIV/AIDS and use of mandatory premarital HIV testing as a prerequisite for marriages among religious leaders in Sokoto, North Western Nigeria. Pan Afr Med J. 2012;11:27. 
25. Adejuyigbe EA, Fasubaa OB, Onayade AA. Sociodemographic characteristics of HIV-positive mother-child pairs in Ile-Ife, Nigeria. AIDS care. 2004;16(3):275-282

26. Oshi DC, Nakalema S, Oshi LL. Cultural and social aspects of HIV/ AIDS sex education in secondary schools in Nigeria. J Biosoc Sci. 2005;37(2):175-183.

27. Kalichman SC. Preventing AIDS: A Sourcebook for Behavioral Interventions. New Jersey, Lawrence Erlbaum Associates. 1998.
28. Prochaska JO, Velicer WF, Rossi JS, et al. Stages of change and decisional balance for twelve problem behaviors. Health Psychol. 2008;13(1):39-46.

29. Okoror TA, Falade CO, Olorunlana A, et al. Exploring the cultural context of HIV stigma on antiretroviral therapy adherence among people living with HIV/AIDS in southwest Nigeria. AIDS Patient Care STDS. $2013 ; 27(1): 55-64$. 